\title{
Fibrotic Tissue and Middle Turbinate Exhibit Similar Mechanical Properties. Is Fibrosis a Solution in Nasal Polyposis?
}

\author{
Luciano Gregório $^{1}$ Rogério Pezato ${ }^{1}$ Rafael Souza Felici ${ }^{1} \quad$ Eduardo Macoto Kosugi ${ }^{1}$ \\ ${ }^{1}$ ENT Research Laboratory, Department of ENT, Universidade Federal \\ de São Paulo, São Paulo, SP, Brazil \\ Address for correspondence Rogerio Pezato, MD, Department of ENT, \\ Universidade Federal de Sao Paulo, Rua Pedro de Toledo, Sao Paulo, Sao \\ Paulo 04023-900, Brazil (e-mail: pezatobau@ig.com.br). \\ Int Arch Otorhinolaryngol 2017;21:122-125.
}

\begin{abstract}
Introduction Nasal polyposis (NP) is a chronic inflammatory condition of the upper airway characterized by overgrowth of nasal mucosa. Recent studies have shown a mechanical dysfunction in the nasal polyp tissue.

Objective This study aims to evaluate the mechanical properties of nasal fibrotic tissue.

Method This study was an institutional review board approved translational study in 20 participants (8 patients with NP, 7 patients with nasal synechiae, and 5 subjects without sinus disease (control group). We used Controlled Disc Stimulation equipment to compare the curve Pressure/Volume created during the saline solution infusion.

Results The increase of pressure in response to solution injection was lower in the nasal polyp group when compared with control middle turbinate group and fibrotic group. No significant difference was found in the pressure response during solution injection between fibrotic group and control middle turbinate group.

Inferior turbinate group showed significant difference when compared with control middle turbinate group.

Keywords

- nasal polyps

- mucosa

- sinusitis

Conclusion The mechanical dysfunction found in the nasal mucosa of patients with NP provides new insight into this condition. These data allow the belief that the fibrosis has a potential role in increasing interstitial hydrostatic pressure and, consequently, mitigating edema formation in NP.
\end{abstract}

\section{Introduction}

Nasal polyposis (NP) is a chronic inflammatory condition of the upper airway characterized by overgrowth of nasal mucosa caused by the influx of inflammatory cells. Patients with NP experience a severe imbalance in immunomodulation ${ }^{1}$ and an abnormal remodeling process, characterized by low production of transforming growth factor- $\beta$ (TGF- $\beta$ ) and a lack of collagen, when compared with healthy subjects. ${ }^{2,3}$ Similar results have been found for the proteoglycans biglycan, lumican, ${ }^{4}$ tenascin, and fibronectin. ${ }^{5}$ The altered composition of extracellular matrix in NP creates a mechanical dysfunction in the nasal mucosa, which impairs the interstitial hydrostatic response during interstitial extravasation of volume in polypoid tissue. ${ }^{6}$

During the inflammatory process the balance between oncotic and hydrostatic pressure [(capillary osmotic pressure - interstitial osmotic pressure) - (hydrostatic interstitial pressure - hydrostatic capillary pressure)] is critical to limit edema formation. Given that the nasal polypoid tissue does not increase the interstitial hydrostatic pressure received

July 26, 2016

accepted

September 6, 2016

published online

October 26, 2016
DOI http://dx.doi.org/

10.1055/s-0036-1593728. ISSN 1809-9777.
Copyright $(2017$ by Thieme-Revinter

Publicações Ltda, Rio de Janeiro, Brazil
License terms

() (1) $\Theta \circledast$ 
properly, as found in different inflamed tissues, a greater volume is necessary to achieve the balance between the pressures (the polyp requires three times more infusion of saline solution to reach the same interstitial hydrostatic pressure found in healthy middle meatus mucosa). ${ }^{6}$

The interstitial hydrostatic pressure response depends on the tissue mechanical properties which in turn are related to the extracellular matrix.

Fibrotic tissue is characterized by high concentration of collagen. In this context, it should be expected that variations in the extracellular matrix could have an impact on tissue mechanical properties and, consequently, on interstitial hydrostatic pressure response.

The objective of this manuscript aims to evaluate the mechanical properties of nasal fibrotic tissue and compare it to nasal polypoid tissue and healthy middle meatus mucosa.

\section{Material and Methods}

\section{Study Population}

We selected participants among the patients of the Rhinology Section between 2014 and 2015. The study population was formed by 8 patients with NP, 7 patients with nasal synechiae, and 5 controls without nasal synechiae or sinus disease. All participants underwent an otorhinolaryngological assessment including nasal endoscopy.

\section{Inclusion Criteria}

European Position Paper on Rhinosinusitis and Nasal Polyps (EPOS) 2012 NP definition for research (presence of two or more symptoms, visualization of polyps in both middle meatus at nasal endoscopy, and confirmation by CT-scans) was used in this study as presence of NP. ${ }^{7}$

We considered nasal synechiae when endoscopically diagnosed and confirmed by palpation with a Cottle elevator, regardless of symptoms.
Control group was formed by patients submitted to septoplasty/turbinectomy, without sinus alterations at CT-scans or nasal synechiae under endoscopic visualization.

\section{Exclusion Criteria}

We excluded patients with age below 18 years old from the study.

\section{Study Design}

The local ethics committee approved this study under protocol number 01510612.8.0000.5505.

After observing inclusion and exclusion criteria, this study included 8 patients with NP, 7 patients with nasal synechiae, and 5 controls subjects without nasal synechiae or sinus disease. The main measurement of this study was the submucosal local pressure. Thereby, we formed four groups: Nasal Polyps, Synechiae, Middle Turbinate, and Inferior Turbinate.

We measured the pressures within the submucosa during infusion of 0.2 to $1 \mathrm{ml}$ of saline solution, presenting data as mean pressure /volume plots ( - Fig. 1 - illustration of a single measurement). A 22-gauge needle coupled to a Controlled Disc Stimulation device (CDS, Smith \& Nephew, Memphis, TN, USA) was used to inject the controlled volume within the submucosa to measure local pressure. ${ }^{6}$ All patients were under general anesthesia, and received no intranasal drugs before the saline injection.

\section{Statistical Analysis}

We analyzed study data in PASW Statistics for Windows, Version 18 (SPSS Inc., Chicago, IL, USA). We used the MannWhitney $U$ test to evaluate between-group differences. P values of $<0.05$ were deemed significant.

\section{Results}

There was no difference in age between the synechiae and NP groups $(p=0.9)$. We found a significant difference in age between the control group and the NP group $(p=0.04)$.
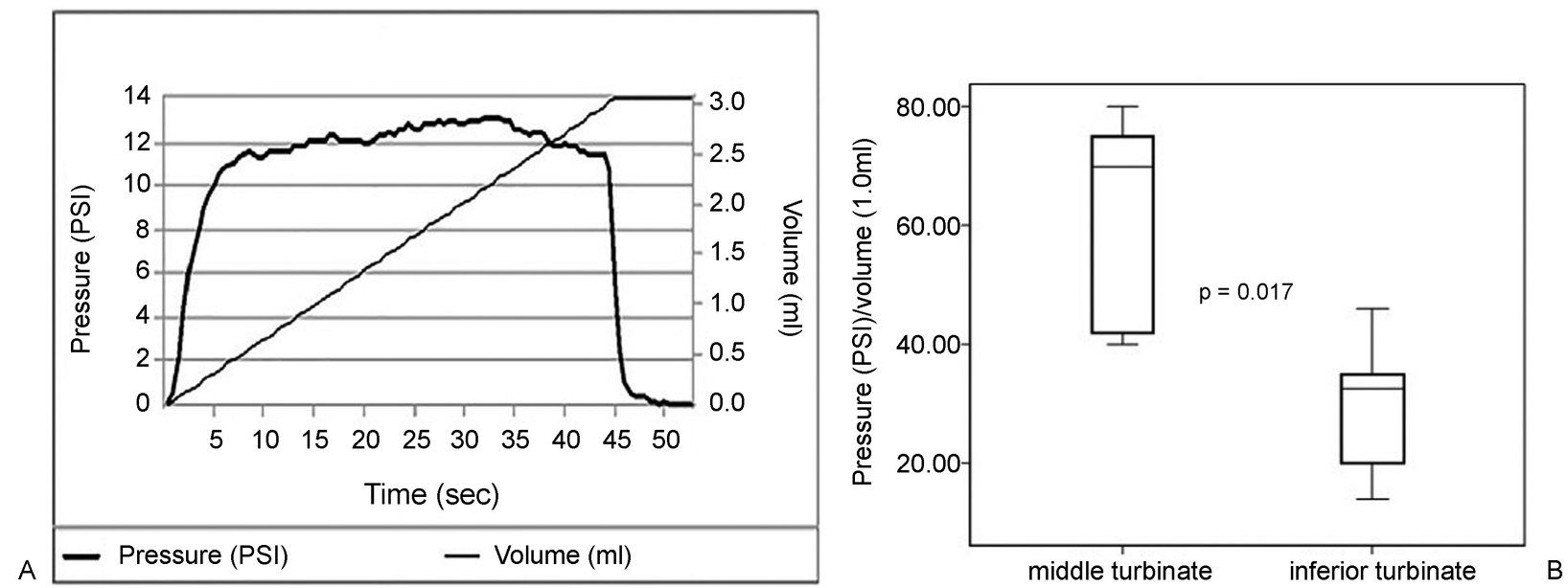

Fig. 1 (A) Graphic illustration of increase in interstitial pressure during volume infusion in a single patient. (B) Comparison of pressure increase in healthy middle turbinate versus healthy inferior turbinate after saline infusion. The pressure increase in the middle turbinate is greater than in the inferior turbinate. 


\section{Increase in Pressure versus Volume (PSI/ml)}

The first part of the pressure-volume $(\mathrm{P} / \mathrm{V})$ plot demonstrated linear variation ( - Fig. 1A); we therefore used the mean $\mathrm{P} / \mathrm{V}$ to compare the hydrostatic pressure created during infusion.

We observed that the mean $\mathrm{P} / \mathrm{V}$ was lower in the submucosa of polypoid tissue than in the submucosa of middle turbinates ( - Fig. 2A) and fibrotic tissue ( - Fig. 2B). We found no significant difference in mean $\mathrm{P} / \mathrm{V}$ between fibrotic tissue and healthy middle turbinate mucosa ( $\mathbf{F i g . ~ 2 C ) . ~}$

When we compared turbinates in the control group, we found a lower mean $\mathrm{P} / \mathrm{V}$ in inferior turbinates as compared

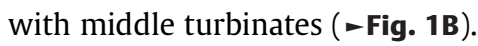

\section{Discussion}

The mechanical dysfunction found in the nasal mucosa of patients with NP provides new insight into this condition. In this study, the concept of NP is more focused on the consequences caused by the effects of a chronic inflammation in patients prone to developing NP than on understanding the different types of inflammatory process involved (orchestrated by Th1 or Th2, predominance of eosinophils or neutrophils).

Consequently, new forms of treatment can be suggested. The interstitial hydrostatic pressure (IHP) during volume extravasation is lower in patients with NP than in healthy nasal mucosa, creating an imbalance in the pressures (osmotic and hydrostatic) responsible for water return into the capillaries.

In theory, mechanisms that increase the IHP could partially prevent edema formation in NP, and one plausible hypothesis would be to shift the remodeling process in a more fibrotic direction. To evaluate this hypothesis, we investigated the IHP response during volume infusion into nasal tissues.

Our finding of higher pressure in the middle turbinate from healthy nasal mucosa than in nasal polypoid tissue corroborates a previous study. ${ }^{6}$

The higher IHP found in fibrotic tissue (synechiae) after saline infusion when compared with polypoid nasal mucosa
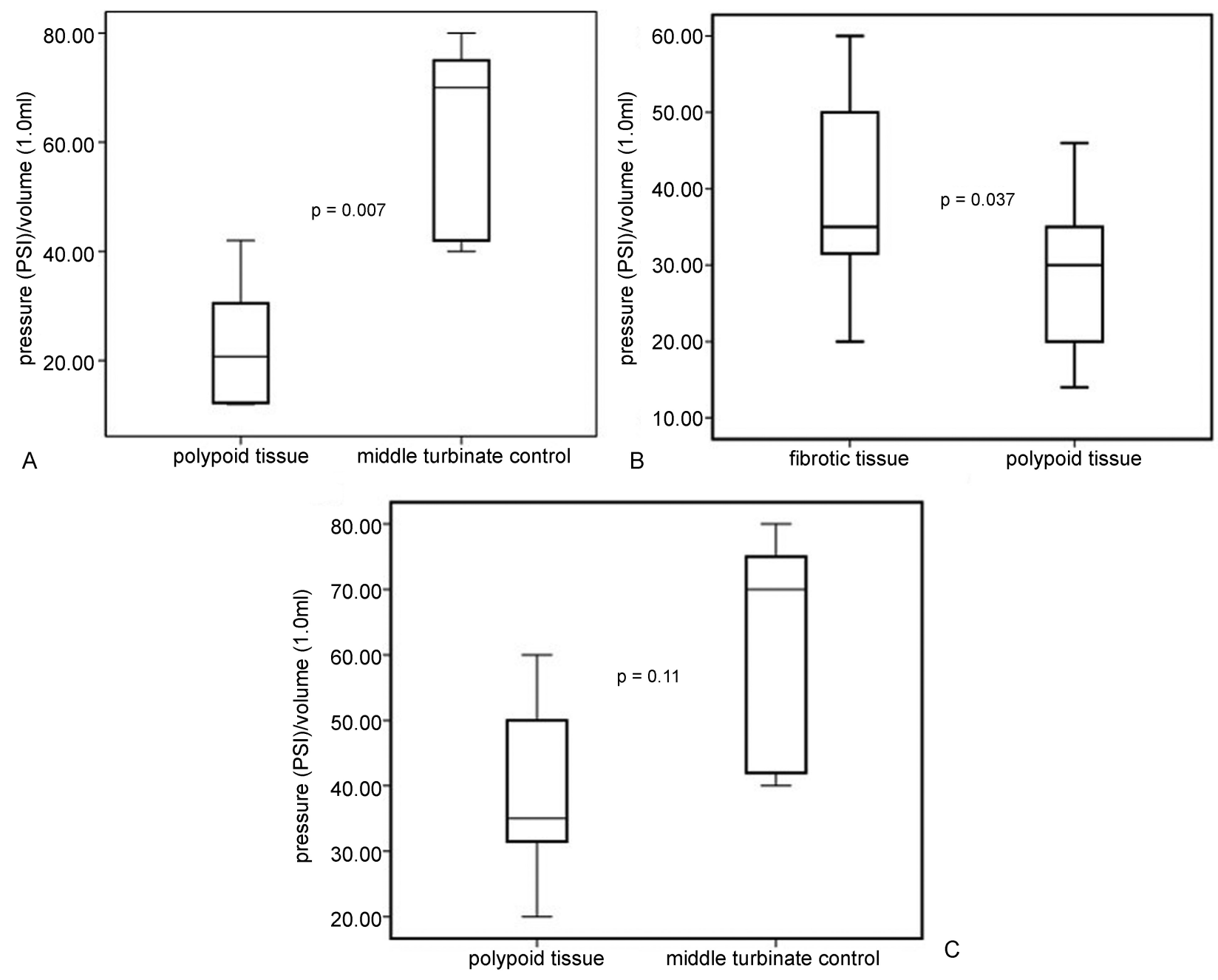

C

Fig. 2 (A) Comparison of pressure increase in nasal polypoid tissue versus healthy nasal mucosa (middle turbinate), the pressure increase is greater in healthy middle turbinate than in polypoid tissue. (B) Comparison of pressure increase in fibrotic tissue (synechia) versus nasal polypoid tissue, the pressure increase is greater in fibrotic tissue than in polypoid tissue. (C) Comparison of pressure increase in fibrotic tissue (synechia) versus healthy nasal mucosa (middle turbinate). There is no significant difference in pressure. 
supports, from a mechanical standpoint, the typical histological findings (high concentration of extracellular matrix, especially collagen, in fibrotic tissue versus soft tissue with a lack of extracellular matrix in NP). 2,4,5,8,9

The mechanical properties found in fibrotic tissue seem to be more similar to healthy nasal mucosa than to nasal polypoid tissue (-Fig. 2), making fibrosis a potential option to interfere with the abnormal remodeling process found in NP.

Although we found a significant difference in age between the control group and the NP group, our focus in this experiment was to assess the fibrotic tissue. The Synechiae group (fibrotic tissue) did not have a significant difference in age when compared with Nasal Polyps group and control groups (Middle Turbinate and Inferior Turbinate group).

Finally, our data provide further support for the notion that the inferior turbinate is a different structure from the middle turbinate (-Fig. 1B). ${ }^{10}$ Other authors have already demonstrated this fact in terms of histology, ${ }^{11}$ airflow physiology, ${ }^{12}$ and nasal nitric oxide levels. ${ }^{13}$ In view of these findings, data generated through the use of inferior turbinate tissue as control in NP research should be analyzed very carefully.

We believe that fibrosis has a potential role in increasing IHP and, consequently, mitigating edema formation in NP.

\section{Conclusion}

Fibrotic tissue exhibits a greater increase in IHP in response to acute volume infusion than nasal polypoid tissue. This increase of IHP in fibrotic tissue closely resembles the response seen in healthy nasal mucosa, suggesting that a tissue remodeling process prone to a more fibrotic direction could slow down the NP progression.

\section{Conflict of Interest}

The authors declare no conflict of interest.

\section{References}

1 Pezato R, de Almeida DC, Bezerra TF, et al. Immunoregulatory effects of bone marrow-derived mesenchymal stem cells in the nasal polyp microenvironment. Mediators Inflamm 2014;2014:583409

2 Van Bruaene N, Derycke L, Perez-Novo CA, et al. TGF-beta signaling and collagen deposition in chronic rhinosinusitis. J Allergy Clin Immunol 2009;124(2):253-259, 259.e1-259.e2

3 Balsalobre L, Pezato R, Perez-Novo C, et al. Epithelium and stroma from nasal polyp mucosa exhibits inverse expression of TGF- $\beta 1$ as compared with healthy nasal mucosa. J Otolaryngol Head Neck Surg 2013;42:29

4 Lee $\mathrm{SH}$, Park JH, Oh BH, et al. Analysis of proteoglycan gene messages in human nasal mucosa and nasal polyp using dot blot hybridization. Acta Otolaryngol 2001;121(3):398-402

5 Liu Z, Gao Q, Zhang S, You X, Cui Y. [Expression of tenascin and fibronectin in nasal polyps]. Zhonghua Er Bi Yan Hou Ke Za Zhi 2002;37(3):173-176

6 Pezato R, Voegels RL, Pinto Bezerra TF, Perez-Novo C, Stamm AC, Gregorio LC. Mechanical disfunction in the mucosal oedema formation of patients with nasal polyps. Rhinology 2014;52(2):162-166

7 Fokkens WJ, Lund VJ, Mullol J, et al. EPOS 2012: European position paper on rhinosinusitis and nasal polyps 2012. A summary for otorhinolaryngologists. Rhinology 2012;50(1):1-12

8 Yang YC, Zhang N, Van Crombruggen K, Hu GH, Hong SL, Bachert C. Transforming growth factor-beta1 in inflammatory airway disease: a key for understanding inflammation and remodeling. Allergy 2012;67(10):1193-1202

9 Pezato R, Voegels RL. Why do we not find polyps in the lungs? Bronchial mucosa as a model in the treatment of polyposis. Med Hypotheses 2012;78(4):468-470

10 Pezato R, Voegels RL, Stamm AC, Gregório LC. Why we should avoid using inferior turbinate tissue as control to Nasal Polyposis studies. Acta Otolaryngol 2016;136(9):973-975

11 Berger G, Finkelstein Y, Ophir D, Landsberg R. Old and new aspects of middle turbinate histopathology. Otolaryngol Head Neck Surg 2009;140(1):48-54

12 Wang Y, Lee HP, Gordon BR. Impacts of fluid dynamics simulation in study of nasal airflow physiology and pathophysiology in realistic human three-dimensional nose models. Clin Exp Otorhinolaryngol 2012;5(4):181-187

13 Takeno S, Yoshimura H, Kubota K, Taruya T, Ishino T, Hirakawa K. Comparison of nasal nitric oxide levels between the inferior turbinate surface and the middle meatus in patients with symptomatic allergic rhinitis. Allergol Int 2014;63(3):475-483 